\title{
Impression Materials
}

By Leanna Wynne MA RDH FHEA

University of Portsmouth Dental Academy

Principal Lecturer and Associate Head (Education)

Leanna Wynne

University of Portsmouth Dental Academy

William Beatty Building

Hampshire Terrace

Portsmouth PO1 2QG

Tel; 02392845379

Email; leanna.wynne@port.ac.uk

The aim of the article is to provide a brief guide to Dental Impression materials 


\section{Impression Materials}

\section{Introduction}

Depending on the clinical situation, choosing the right impression material is one component in achieving an excellent clinical outcome for the patient. To accurately reproduce a model of the teeth and alveolar ridges impression materials are used to create a negative mould that is then used to make a cast (copy) of the dentition. A cast may be used for treatment planning, the construction of dentures, crowns or bridges and for orthodontic work. More complex dental work might require the use of impression materials for maxillofacial surgery cases. There are many types of impression materials to select from, each with their own advantages and disadvantages and they are chosen on the basis of relative need for accuracy, dimensional stability and elasticity. This article will first provide a brief overview of the role of the dental nurse when using impression materials and then consider the types of dental impression materials available for use. An outline of the disinfection process of impressions will then follow.

\section{The role of the dental nurse}

The General Dental Council (GDC) requires that on qualification a Dental nurse (DN) is able to describe the commonly used dental biomaterials and their application and that they are able to prepare, mix and handle dental materials (General Dental Council 2013). Stated within the Scope of Practice document published in September 2013 by the GDC a DN may also choose to develop professionally and learn to pour, cast and trim study models. Additionally a DN may develop further skills carried out on prescription from, or under the direction of, another registrant, taking impressions (where appropriate), constructing occlusal registration rims and special trays and constructing mouthguards and bleaching trays. Prior to carrying out any of these additional duties a DN should ensure that they have undertaken the necessary education and training to ensure competence. In addition a DN is advised to check that they are indemnified to carry out the additional skills that they have developed.

\section{Properties of impression materials}

Accuracy and dimensional stability - it is important that an impression material can accurately replicate and preserve intraoral surface details and dimensions until a working cast can be poured. Any distortion can result in an ill-fitting prosthesis. Tear resistance - the ability to remain intact on removal from the oral cavity, as tears would reduce the accuracy.

Patient tolerance of material - once in the mouth the setting time should be relatively quick to reduce patient discomfort. The taste would ideally be acceptable to the patient and the consistency fluid enough to be placed in the oral cavity prior to setting, but not too fluid that it would run from the impression tray.

Ease of use by operator - a material that is easy to mix and handle with, ideally, a long working time and a short setting time. The working time of a material is typically determined by the room temperature and the setting time by the temperature 
and moisture in the oral cavity. Clinicians are advised to read the Manufacturers instructions when working with an unfamiliar material.

Inexpensive - an economically viable product with a long shelf life can benefit the practice and the patient in relation to cost. The long shelf life may not always be possible and some materials might need to be stored in a refrigerator to maintain the shelf life. It is advised to always check the manufacturers instructions.

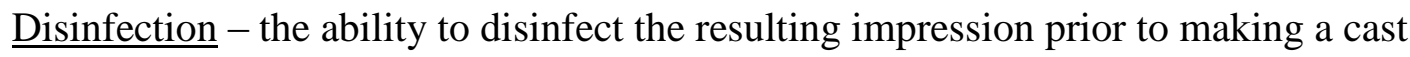
without affecting the accuracy or dimensional stability of the material.

\section{Classification of impression materials}

Impression materials can be classified by their key property; rigid, water-based or elastomers.

\section{$\underline{\text { Rigid materials }}$}

These include impression plaster, impression compound and zinc oxide eugenol (ZOE). Impression plaster was the first material used for edentulous and dentate impressions, however, due to the rigid setting impression plaster would only now be used for edentulous cases where there are no undercuts. ZOE impression paste would also be used in this way, although it is tenacious by nature and can adhere to tissues ZOE has good dimensional stability. Impression compound is supplied as sheets or sticks. The material will distort over undercuts and is therefore best used for single tooth impressions where there are no undercuts.

\section{$\underline{\text { Water-based materials }}$}

Alginate (derived from seaweed) is an irreversible hydrocolloid and Agar-Agar is a reversible hydrocolloid. Both are composed of approximately $85 \%$ water making them inherently unstable materials, as water from the material can be lost to the environment (syneresis) causing shrinkage, or, water can be absorbed by the material from the air (imbibition). Hydrocolloid impression materials were first developed in the 1920's. Alginate is described as being an irreversible hydrocolloid as it cannot return to a solution state once set. It is a common impression material used in dentistry as it is inexpensive, easy to use and is usually tolerated well by the patient. However, alginate has weak dimensional stability and is generally wrapped in wet gauze once the impression is taken and it is recommended that a cast be poured within 30 minutes when using conventional alginate or as soon as possible to minimise any distortion. Extended storage alginate is also available and claims to offer dimensional stability for up to 100 hours (Walker, Burchhard, Mitts and Williams, 2010). Alginate is mainly used for applications where a reduction in accuracy can be accommodated. The material is still usually mixed by hand and relies on the operator achieving an accurate powder/water ratio and skilful mixing to reduce the possibility of inaccuracies.

\section{$\underline{\text { Elastomeric materials }}$}


Classification of elastomeric impression materials can be based on viscosity and on chemistry. The material can be heavy, regular or light in viscosity, referring to the ability of the material to flow around the tissues, additionally some are made available as very heavy (putty consistency) or ultra light. A light viscosity will flow well into areas to provide detail where a heavier viscosity may not flow. The heavier viscosities are often used to fill the impression tray and a light viscosity material may be placed over the heavier viscosity material to increase the accuracy of the impression. The chemistry of elastomeric materials differ, however, they can be classified simply as rubbery polymers that include polysulphides, silicones, polyethers (PE) and vinyl polysiloxane (VPS). PE and VPS are more commonly used in modern dentistry and vinyl-polyether hybrids (VPES) are also available. A base paste and catalyst are dispensed and mixed together resulting in a chemical reaction that forms a polymer.

Polysulphides were first developed in 1950 and are a highly flexible material usually used for crown and bridge work as they have good dimensional stability with excellent tear strength and provide a high level of accuracy, however, they can be messy to work with and are used only used in special trays. The material is supplied as two pastes (one base and one activator) and mixed in a 1:1 ratio. As a hydrophobic material a dry field is required for accurate results, which is not always easy in a patients mouth, and although dimensionally stable it is advised that the impressions be cast within 24 hours.

Silicone materials are hydrophobic and more rigid than polysulphide materials. The material has a reasonable level of accuracy if cast within 30 minutes of taking the impression but has poor to adequate tear strength and poor dimensional stability. The material can be used in both stock and special trays. On occasion it can be difficult to remove the impression from the mouth of the patient as the material can break on removal. Conventional silicones were first developed in 1955 and are available as heavy, medium or light viscosity. Storage in a refrigerator can extend the shelf life.

Polyether (PE) can be purchased as a conventional impression material and as a light cured PE. Conventional PE was first developed in 1965. The light cure option requires transparent trays and provides operator control over working and setting time. PE is often chosen for crown and bridge procedures due to the level of accuracy attained. PE is the most rigid of the elastomeric materials reducing the tear strength, however, the elasticity has improved over the years and in particular in the light cure version. When immersed in water a hydrophilic material will absorb the water and swell. PE materials are inherently hydrophilic resulting in the surface moisture being absorbed on close contact with oral tissues and simultaneously registering an accurate impression.

Vinyl polysiloxane (VPS) may also be referred to as an addition silicone and was first developed in 1975. VPS is often the material of choice for crown and bridge work 
due to its ease of use, accuracy and dimensional stability. Hydrophobic by nature some VPS materials have been developed (since 1986) to incorporate surfactants that provide hydrophilic properties. This then allows for increased accuracy over moist surfaces within the oral cavity, although hydrophilic VPS may not displace excess saliva effectively.

\section{Disinfection of dental impressions}

Prior to impressions being sent out of the practice the impression is to be disinfected to prevent the transmission of organisms from the oral cavity that may reside on the material. The process of disinfection can raise issues as some materials are water based, for example alginate, and some are prone to water uptake or loss, for example PE. The surface of the cast might also be affected by the disinfectant used on the impression. The impression surface should first be rinsed to remove the more obvious contaminants under running water until visibly clean. The impression should then be disinfected according to the manufacturers instructions. The disinfection agents can be sprayed on or the impression can be immersed in the solution, the latter proving more reliable as it ensures that all surfaces are disinfected (Almortadi and Chadwick, 2010). Following disinfection the impression is then washed thoroughly in water once again. When sending the impression out of the practice, for example to a laboratory, details of the disinfection process are to be indicated on a form that is then attached to the impression packaging (Department of Health, 2013).

\section{Conclusion}

An ideal impression material would be smooth flowing, hydrophilic, resistant to tearing and one that does not shrink when setting, however, it is the clinical situation that will determine the selection of the final impression material coupled with the cost of the material. There is no one product that will meet the requirements of all clinical situations, therefore a practice may need to stock up to three different types of impression materials. It is important to follow the manufacturers instructions on the use and the disinfection of the impression material to maximise the accuracy and dimensional stability. Impression taking is a weekly, if not daily task within a practice and an understanding of the chemistry behind the material can help when choosing the right impression material for a given clinical situation.

\section{References:}

Almortadi, N., and Chadwick, R.G. (2010). Disinfection of dental impressions compliance to accepted standards. British Dental Journal. 209:12, 607- 611.

Department of Health. (2013). Decontamination Health Technical Memorandum 0105: Decontamination in primary care dental practices. London: DoH

General Dental Council. (2013). Preparing for Practice. Dental team learning outcomes for registration. [Electronic Version]. London: GDC 
General Dental Council. (2013). Scope of Practice. [Electronic Version]. London: GDC

Walker, M., Burckhard, J., Mitts, D.A., and Williams, K.B. (2010). Dimensional change over time of extended-storage alginate impression materials. Angle Orthodontist. 80:6, 1110-1115 